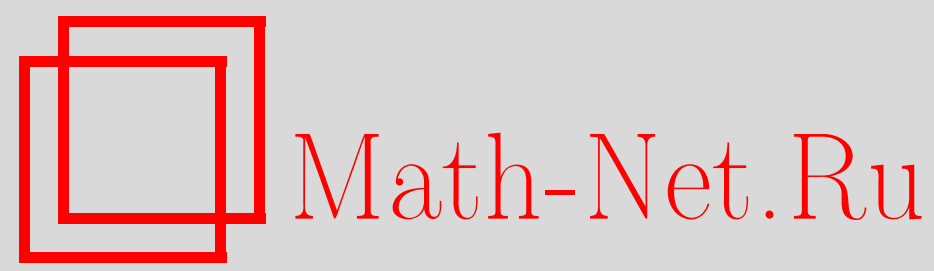

А. Б. Рагимов, K численному решению обратных задач для линейного параболического уравнения, Вестник ТвГУ. Серия: Прикладная математика, 2018, выпуск 1, 69-87

DOI: https://doi.org/10.26456/vtpmk162

Использование Общероссийского математического портала Math-Net.Ru подразумевает, что вы прочитали и согласны с пользовательским соглашением

http://www.mathnet.ru/rus/agreement

Параметры загрузки:

IP: 54.80 .73 .141

26 апреля 2023 г., $14: 14: 15$ 
УДК 519.633

\section{К ЧИСЛЕННОМУ РЕШЕНИЮ ОБРАТНЫХ ЗАДАЧ ДЛЯ ЛИНЕЙНОГО ПАРАБОЛИЧЕСКОГО УРАВНЕНИЯ}

Рагимов А.Б.

Институт систем управления НАН Азербайджана, г. Баку

Поступила в редакцию 21.09.2017, после переработки 01.03.2018.

В статье рассматриваются обратные задачи для линейного параболического уравнения с неизвестными коэффициентами в правой части. $\mathrm{K}$ данным задачам, в частности, приводятся краевые задачи, с нелокальными условиями. Отдельно рассмотрены случаи, когда идентифицируемые коэффициенты зависят либо только от временной переменной, либо только от пространственной координаты. Предлагается методика численного решения задач с использованием метода прямых. Приводятся результаты численных экспериментов, проведенных на тестовых задачах.

Ключевые слова: обратная задача, нелокальные условия, метод прямых, параболическое уравнение, параметрическая идентификация.

Вестник ТвГУ. Серия: Прикладнал математика. 2018. № 1. С. 69-87. https://doi.org/10.26456/vtpmk162

\section{Введение}

В последние годы возросло внимание исследователей к коэффициентнообратным задачам относительно процессов, описываемых уравнениями с частными производными, в частности, параболическими уравнениями [1-20]. С подобными задачами приходится сталкиваться на этапе параметрической идентификации математических моделей объектов с распределенными параметрами.

В данной работе исследуется численное решение обратных задач, в которых идентифицируемые коэффициенты зависят лишь от одной переменной: пространственной или временной. $\mathrm{K}$ исследуемым обратным задачам, в частности, приводит важный класс краевых задач с нелокальными условиями [17-26]. Нелокальность условий обусловлена невозможностью на практике проводить замеры измеряемых параметров состояния объекта (процесса) мгновенно или в его отдельно взятых точках. Подобные задачи возникают при изучении явлений, происходящих в плазме [22], процессов распространения тепла $[17,23,24]$ и влагопереноса в капиллярно-пористых средах [25], математическом моделировании технологического процесса внешнего геттерирования при очищении кремниевых плат от примесей [20], в задачах математической биологии [21] и демографии [26].

Исследованию различных классов обратных задач, а именно полученных необходимых условий, а иногда и достаточных условий существования, единственности решения коэффициентно-обратных задач посвящены работы [12-14]. Отметим, 
что эти исследования в настоящее время активно продолжаются в особенности в направлении изучения отдельных классов обратных задач [1-3] в рамках математического моделирования функционирования конкретных процессов, объектов с распределенными параметрами.

Наиболее часто применяемым подходом к решению обратных задач параметрической идентификации является сведение их к задачам минимизации функционала, характеризующего меру отклонения модельных и наблюдаемых выходных величин процесса. Далее для решения соответствующих вариационных задач используются численные методы оптимального управления, приводящие к построению итерационных процедур [9-11].

Другой подход заключается в использовании построения фундаментального решения задачи и приведения ее к интегральному уравнению. В случае, когда участвующие в задаче функции имеют общий вид, использование такого подхода препятствуют ряд трудностей [6, 15-17].

В настоящей статье предлагается подход к численному решению обратных задач для линейных параболических уравнений. Специфика рассматриваемых классов обратных задач заключается, в том, что, во-первых, восстанавливаемые коэффициенты находятся при свободном члене, и, во-вторых, они зависят или только от временной, или только от пространственной координаты. Эта специфика позволяет с помощью метода прямых [27-29] свести решение исходных задач к решению специально построенных задач Коши [30] относительно системы обыкновенных дифференциальных уравнений. Таким образом, в предлагаемым в работе подходе не используются какие-либо итерационные процедуры. В настоящей статье приводятся результаты численных экспериментов и их анализ.

\section{1. Постановка задач}

Рассмотрим коэффициентно-обратную задачу относительно параболического уравнения:

$$
\begin{array}{r}
\frac{\partial v(x, t)}{\partial t}=a(x, t) \frac{\partial^{2} v(x, t)}{\partial x^{2}}+a_{1}(x, t) \frac{\partial v(x, t)}{\partial x}+a_{2}(x, t) v(x, t)+f(x, t)+F(x, t), \\
(x, t) \in \Omega=\{(x, t): 0<x<l, 0<t \leq T\},
\end{array}
$$

где

$$
F(x, t)=\sum_{s=1}^{L} B_{s}(x, t) C_{s}(x),
$$

при следующих начально-краевых условиях и дополнительных условиях:

$$
\begin{gathered}
v(x, 0)=\phi_{0}(x), \quad x \in[0, l], \\
v(0, t)=\psi_{0}(t), \quad v(l, t)=\psi_{1}(t), \quad t \in[0, T], \\
v\left(x, \bar{t}_{s}\right)=\phi_{1 s}(x), \quad x \in[0, l], \bar{t}_{s} \in(0, T], \quad s=1, \ldots, L .
\end{gathered}
$$

Здесь $L>0$ - заданное целое число, $\bar{t}_{s} \in(0, T], s=1, \ldots, L-$ заданные моменты времени; заданные функции $a(x, t)>0, a_{1}(x, t), a_{2}(x, t), f(x, t), B_{s}(x, t)$, $\phi_{0}(x), \psi_{0}(t), \psi_{1}(t), \phi_{1 s}(x), s=1, \ldots, L$ равномерно непрерывны и ограничены по 
$x$ и $t ; B_{s}(x, t)$ - линейно-независимые функции, дифференцируемые по $t$, причем $a_{2}(x, t) \leq 0, B_{s}(x, t)>0, \frac{\partial B_{s}(x, t)}{\partial t} \geq 0$. Функции $\phi_{0}(x), \phi_{1 s}(x), \psi_{0}(t), \psi_{1}(t)$ удовлетворяют условиям согласования:

$\phi_{0}(0)=\psi_{0}(0), \quad \phi_{0}(l)=\psi_{1}(0), \quad \phi_{1 s}(0)=\psi_{0}\left(\bar{t}_{s}\right), \quad \phi_{1 s}(l)=\psi_{1}\left(\bar{t}_{s}\right), s=1, \ldots, L$.

Задача (1)-(5) заключается в определении неизвестной непрерывной $L$-мерной вектор-функции $C(x)=\left(C_{1}(x), \ldots, C_{L}(x)\right)^{*}$ и соответствующего решения краевой задачи $v(x, t)$ - дважды непрерывно дифференцируемой по $x$ и один раз непрерывно дифференцируемой по $t$ при $(x, t) \in \Omega$, удовлетворяющих условиям (1)-(5). Известно, что при сделанных выше предположениях обратная задача (1)-(5) имеет решение, причем оно является единственно $[12,13]$.

Возможен случай коэффициентно-обратной задачи, когда в уравнении (1) функция $F(x, t)$ имеет вид

$$
F(x, t)=\sum_{s=1}^{L} C_{s}(x, t) B_{s}(t),
$$

где функции $C_{s}(x, t)$ - заданы, коэффициенты $B_{s}(t)$ идентифицируются, а вместо дополнительного условия (5) может быть задано, например, условие

$$
v\left(\bar{x}_{s}, t\right)=\psi_{2 s}(t), \quad \bar{x}_{s} \in(0, l), \quad t \in[0, T], \quad s=1, \ldots, L .
$$

K частному случаю задачи (1)-(5) приводится следующая краевая задача с нелокальным (интегральным) начальным условием:

$$
\begin{gathered}
\frac{\partial u(x, t)}{\partial t}=a(x) \frac{\partial^{2} u(x, t)}{\partial x^{2}}+a_{1}(x) \frac{\partial u(x, t)}{\partial x}+a_{2}(x) u(x, t)+\tilde{f}(x, t),(x, t) \in \Omega, \\
k_{1} u(x, 0)+\int_{0}^{T} e^{k \tau} u(x, \tau) d \tau=\phi_{0}(x), x \in[0, l], \\
u(0, t)=\tilde{\psi}_{0}(t), u(l, t)=\tilde{\psi}_{1}(t), \quad t \in[0, T],
\end{gathered}
$$

где $k, k_{1}$ - заданные постоянные, $\tilde{f}(x, t), \phi_{0}(x), \tilde{\psi}_{0}(t), \tilde{\psi}_{1}(t)$ - заданные функции. Некоторые частные случаи задачи (8)-(10) рассматривались, например, в работах $[18,19]$.

Для сведения задачи (8)-(10) к задаче (1)-(5) введем функцию

$$
v(x, t)=k_{1} u(x, 0)+\int_{0}^{t} e^{k \tau} u(x, \tau) d \tau .
$$

Дифференцируя (11) по $t$, имеем

$$
u(x, t)=e^{-k t} \frac{\partial v(x, t)}{\partial t}
$$


Из (9)-(12) получим

$$
\left\{\begin{array}{l}
v(x, 0)=k_{1} \frac{\partial v(x, 0)}{\partial t} \\
v(0, t)=k_{1} \tilde{\psi}_{0}(0)+\int_{0}^{t} e^{k \tau} \tilde{\psi}_{0}(\tau) d \tau=\psi_{2}(t) \\
v(x, T)=\phi_{0}(x), \\
v(l, t)=k_{1} \tilde{\psi}_{1}(0)+\int_{0}^{t} e^{k \tau} \tilde{\psi}_{1}(\tau) d \tau=\psi_{1}(t)
\end{array}\right.
$$

Дифференцируя $u(x, t)$ из (12) один раз по $t$ и дважды по $x$ и подставляя результаты в уравнение (8), после некоторых несложных преобразований получаем

$$
\begin{array}{r}
\frac{\partial^{2} v(x, t)}{\partial t^{2}}-a(x) \frac{\partial^{3} v(x, t)}{\partial t \partial x^{2}}-a_{1}(x) \frac{\partial^{2} v(x, t)}{\partial t \partial x}- \\
-\left(a_{2}(x)+k\right) \frac{\partial v(x, t)}{\partial t}-e^{k t} \tilde{f}(x, t)=0 .
\end{array}
$$

Интегрируя обе части (14) по $t$, получаем равенство с точностью до некоторой произвольной функции $C(x)$ :

$$
\frac{\partial v(x, t)}{\partial t}=a(x) \frac{\partial^{2} v(x, t)}{\partial x^{2}}+a_{1}(x) \frac{\partial v(x, t)}{\partial x}+\left(a_{2}(x)+k\right) v(x, t)+f(x, t)+C(x),
$$

где $f(x, t)=\int_{0}^{t} e^{k \tau} \tilde{f}(x, \tau) d \tau$. Функции $v(x, t), C(x)$ должны удовлетворять уравнению (15) и условиям (13).

Задача (13), (15) отличается от задачи параметрической идентификации (1)-(5) лишь начальным условием при $t=0$.

К виду (1), (3), (4), (6), (7) приводится, например, следующая задача с нелокальным краевым условием:

$$
\begin{gathered}
\frac{\partial u(x, t)}{\partial t}=a(t) \frac{\partial^{2} u(x, t)}{\partial x^{2}}+a_{1}(t) \frac{\partial u(x, t)}{\partial x}+a_{2}(t) u(x, t)+\tilde{f}(x, t),(x, t) \in \Omega \\
u(0, t)=\psi_{2}(t), \int_{0}^{l} e^{k \xi} u(\xi, t) d \xi=\psi_{1}(t), \quad t \in[0, T] \\
u(x, 0)=\tilde{\phi}_{0}(x), \quad x \in[0, l] .
\end{gathered}
$$

Решение задачи вида (16)-(18) рассмотрено, например, в работах $[17,18,26]$.

Для приведения задачи (16)-(18) к виду (1), (3), (4), (6), (7) введем функцию

$$
v(x, t)=\int_{0}^{x} e^{k \xi} u(\xi, t) d \xi .
$$

Дифференцируя (19) по $x$, получаем

$$
u(x, t)=e^{-k x} \frac{\partial v(x, t)}{\partial x}
$$


Дифференцируя (20) один раз по $t$ и дважды по $x$ и подставляя это выражение в (16), после несложных преобразований имеем:

$$
\begin{array}{r}
\frac{\partial^{2} v(x, t)}{\partial t \partial x}=a(t) \frac{\partial^{3} v(x, t)}{\partial x^{3}}+\left(a_{1}(t)-2 k a(t)\right) \frac{\partial^{2} v(x, t)}{\partial x^{2}}+ \\
+\left(k^{2} a(t)-k a_{1}(t)+a_{2}(t)\right) \frac{\partial v(x, t)}{\partial x}+e^{k x} \tilde{f}(x, t)
\end{array}
$$

Интегрируя обе части этого уравнения по $x$, получаем уравнение с точностью до произвольной функции $B(t)$ :

$$
\begin{aligned}
& \frac{\partial v(x, t)}{\partial t}=a(t) \frac{\partial^{2} v(x, t)}{\partial x^{2}}+\left(a_{1}(t)-2 k a(t)\right) \frac{\partial v(x, t)}{\partial x}+ \\
& \quad+\left(k^{2} a(t)-k a_{1}(t)+a_{2}(t)\right) v(x, t)+f(x, t)+B(t)
\end{aligned}
$$

при этом использовано обозначение $f(x, t)=\int_{0}^{x} e^{k \xi} \tilde{f}(\xi, t) d \xi$. Из (17)-(20) имеем следующие начальные и краевые условия:

$$
\begin{gathered}
v(0, t)=0, \quad v(l, t)=\psi_{1}(t), \quad t \in[0, T] \\
v(x, 0)=\phi_{0}(x), \quad x \in[0, l]
\end{gathered}
$$

где

$$
\begin{gathered}
\phi_{0}(x)=\int_{0}^{x} e^{k \xi} \tilde{\phi}_{0}(\xi) d \xi ; \\
\frac{\partial v(0, t)}{\partial x}=\psi_{2}(t), \quad t \in[0, T] .
\end{gathered}
$$

Полученная коэффициентно обратная задача (21)-(24) является частным случаем задачи (1), (3), (4), (6), (7).

\section{2. Решение задач}

В настоящей статье предлагается подход к численному решению задач (1)-(5) и $(1),(3),(4),(6),(7)$, основанный на использовании метода прямых. Например, задача (1)-(5) сводится к системе обыкновенных дифференциальных уравнений с неизвестными параметрами.

В области $\Omega$ проведем прямые $x_{i}=i h_{x}, i=0,1, \ldots, N, h_{x}=l / N$. На этих прямых определим функции:

$$
v_{i}(t)=v\left(x_{i}, t\right), \quad t \in[0, T], i=0,1, \ldots, N,
$$

для которьх, согласно (3)-(5), справедливы следующие условия:

$$
\begin{gathered}
v_{i}(0)=\phi_{0}\left(x_{i}\right)=\phi_{0 i}, i=0, \ldots, N, \\
v_{0}(t)=\psi_{0}(t), \quad v_{N}(t)=\psi_{1}(t), \quad t \in[0, T],
\end{gathered}
$$




$$
v_{i}\left(\bar{t}_{s}\right)=\phi_{1 s}\left(x_{i}\right)=\phi_{1 s, i}, \quad \bar{t}_{s} \in(0, T], \quad s=1, \ldots, L, \quad i=0, \ldots, N .
$$

На прямьг $x=x_{i}$ аппроксимируем производные $\partial v / \partial x, \partial^{2} v / \partial x^{2}$ с использованием центральных разностных соотношений:

$$
\begin{gathered}
\left.\frac{\partial v(x, t)}{\partial x}\right|_{x=x_{i}}=\frac{v_{i+1}(t)-v_{i-1}(t)}{2 h_{x}}+O\left(h_{x}^{2}\right), \quad i=1, \ldots, N-1, \\
\left.\frac{\partial^{2} v(x, t)}{\partial x^{2}}\right|_{x=x_{i}}=\frac{v_{i+1}(t)-2 v_{i}(t)+v_{i-1}(t)}{h_{x}^{2}}+O\left(h_{x}^{2}\right), \quad i=1, \ldots, N-1 .
\end{gathered}
$$

Далее используем обозначения:

$$
\begin{array}{r}
a_{i}(t)=a\left(x_{i}, t\right), \quad \bar{f}_{i}(t)=f\left(x_{i}, t\right), \quad a_{1 i}(t)=a_{1}\left(x_{i}, t\right), \quad a_{2 i}(t)=a_{2}\left(x_{i}, t\right), \\
B_{s i}(t)=B_{s}\left(x_{i}, t\right), \quad C_{s i}=C_{s}\left(x_{i}\right), \quad s=1, \ldots, L, \quad i=1, \ldots, N-1 .
\end{array}
$$

Подставляя (28), (29) в (1), получаем систему обыкновенных дифференциальных уравнений $(N-1)$-го порядка с неизвестным (идентифицируемым) вектором параметров $C_{s}=\left(C_{s 1}, \ldots, C_{s, N-1}\right)^{T}$ :

$$
\begin{array}{r}
v_{i}^{\prime}(t)=\frac{a_{i}(t)}{h_{x}^{2}}\left[v_{i+1}(t)-2 v_{i}(t)+v_{i-1}(t)\right]+\frac{a_{1 i}(t)}{2 h_{x}}\left[v_{i+1}(t)-v_{i-1}(t)\right]+ \\
+a_{2 i}(t) v_{i}(t)+\bar{f}_{i}(t)+\sum_{s=1}^{L} B_{s i}(t) C_{s i}, \quad i=1,2, \ldots, N-1 .
\end{array}
$$

Эту систему с учетом (25), (26) можно записать в векторно-матричном виде:

$$
\begin{gathered}
\dot{v}(t)=A(t) v(t)+f(t)+\sum_{s=1}^{L} E_{s B(t)} C_{s}, t \in(0, T], \\
v(0)=\phi_{0}, \\
v\left(\bar{t}_{s}\right)=\phi_{1 s}, \quad s=1, \ldots, L,
\end{gathered}
$$

где $v(t)=\left(v_{1}(t), \ldots, v_{N-1}(t)\right)^{T}, B_{s}(t)=\left(B_{s 1}(t), \ldots, B_{s, N-1}(t)\right)^{T}$, $\phi_{0}=\left(\phi_{01}, \ldots, \phi_{0, N-1}\right)^{T}, \quad \phi_{1 s}=\left(\phi_{1 s, 1}, \ldots, \phi_{1 s, N-1}\right)^{T}, E_{s B(t)}-(N-1)$-мерная квадратная матрица, в которой $i$-й элемент главной диагонали равен $i$-й компоненте вектора $B_{s}(t)$, т. е. $B_{s i}(t)$, все остальные элементы равны нулю. Ненулевые элементы квадратной $(N-1)$-мерной трехдиагональной матрицы $A$ имеют вид

$$
\begin{gathered}
\tilde{a}_{i i}(t)=\frac{1}{h_{x}^{2}}\left[-2 a_{i}(t)+h_{x}^{2} a_{2 i}(t)\right], \quad i=1, \ldots, N-1, \\
\tilde{a}_{i, i+1}(t)=\frac{1}{h_{x}^{2}}\left[a_{i}(t)+\frac{h_{x}}{2} a_{1 i}(t)\right], i=1, \ldots, N-2, \\
\tilde{a}_{i, i-1}(t)=\frac{1}{h_{x}^{2}}\left[a_{i}(t)-\frac{h_{x}}{2} a_{1 i}(t)\right], \quad i=2, \ldots, N-1 .
\end{gathered}
$$


Вектор $f(t)$ определяется следующим образом:

$$
\begin{aligned}
f(t)=\left(\bar{f}_{1}(t)+\right. & \left(\frac{a_{1}(t)}{h_{x}^{2}}-\frac{a_{11}(t)}{2 h_{x}}\right) \psi_{0}(t), \bar{f}_{2}(t), \ldots, \bar{f}_{N-2}(t), \\
& \left.\bar{f}_{N-1}(t)+\left(\frac{a_{N-1}(t)}{h_{x}^{2}}+\frac{a_{1, N-1}(t)}{2 h_{x}}\right) \psi_{1}(t)\right)^{T} .
\end{aligned}
$$

Задача (30)-(32) при налагаемых условиях на коэффициенты уравнения (1) и функции в начально-краевых условиях аппроксимирует задачу (1)-(5) с точностью $O\left(h_{x}^{2}\right)$ (вопросы сходимости решения задачи (30)-(32) к решению задачи (1)(5) и оценки погрешности рассмотрены в работе [31]). Отметим, что погрешность $O\left(h_{x}^{2}\right)$ можно улучшить за счет использования схем аппроксимации производных по $x$ более высокого порядка $[28,29]$.

Обозначим $0_{(N-1) \times(N-1)}$ нулевую $(N-1)$-мерную квадратную матрицу.

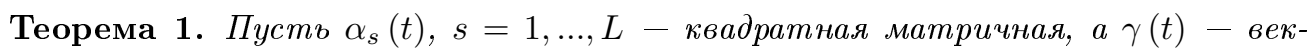
торная функции размерности $(N-1)$ являются решением задач Коши:

$$
\begin{gathered}
\dot{\alpha}_{s}(t)=A(t) \alpha_{s}(t)+E_{s B(t)}, \quad s=1, \ldots, L, \\
\alpha_{s}(0)=0_{(N-1) \times(N-1)}, \quad s=1, \ldots, L, \\
\dot{\gamma}(t)=A(t) \gamma(t)+f(t), \\
\gamma(0)=\phi_{0} .
\end{gathered}
$$

Тогда для произвольного постоянного $(N-1)$-мерного вектора $C_{s}$ решением задачи Коши (30), (31) будет следующая вектор-функция

$$
v(t)=\sum_{s=1}^{L} \alpha_{s}(t) C_{s}+\gamma(t)
$$

Доказательство. С учетом (34), (36) очевидно, что функция $v(t)$, определенная из $(37)$, для произвольного вектора $C_{s} \in R^{N-1}, s=1, \ldots, L$ удовлетворяет начальному условию (31). Дифференцируя обе части равенства (37) с учетом (33), (35), имеем

$$
\begin{array}{r}
\dot{v}(t)=\sum_{s=1}^{L} \dot{\alpha}_{s}(t) C_{s}+\dot{\gamma}(t)=\sum_{s=1}^{L}\left[A(t) \alpha_{s}(t)+E_{s B(t)}\right] C_{s}+[A(t) \gamma(t)+f(t)]= \\
=A(t)\left(\sum_{s=1}^{L} \alpha_{s}(t) C_{s}+\gamma(t)\right)+\sum_{s=1}^{L} E_{s B(t)} C_{s}+f(t)= \\
=A(t) v(t)+\sum_{s=1}^{L} E_{s B(t)} C_{s}+f(t) .
\end{array}
$$

Следовательно, функция $v(t)$ удовлетворяет уравнению (30).

Решив раздельно матричную задачу Коши (33), (34) для определения $\alpha_{s}(t), s=1, \ldots, L$ и задачу Коши $(35),(36)$ относительно векторной функции $\gamma(t)$ и использовав условие (32) и представление $(37)$, получим равенство

$$
v\left(\bar{t}_{s}\right)=\phi_{1 s}=\sum_{s=1}^{L} \alpha_{s}\left(\bar{t}_{s}\right) C_{s}+\gamma\left(\bar{t}_{s}\right), \quad s=1, \ldots, L,
$$


представляющее собой алгебраическую систему уравнений порядка $(N-1)$, из которой можно определить идентифицируемый вектор $C_{s}, s=1, \ldots, L$.

Далее, используя значения компонент вектора $C_{s}=\left(C_{s}\left(x_{1}\right), \ldots, C_{s}\left(x_{N-1}\right)\right)^{T}, s=1, \ldots, L$, и применяя какой-либо метод интерполяции или аппроксимации, можно восстановить искомую функцию $C_{s}(x), s=1, \ldots, L$ на заданном классе функций.

В случае необходимости определения самого решения $v(x, t)$ краевой задачи (1)-(5), достаточно решить задачу Коши (30), (31).

Предложенный подход можно аналогичным образом использовать для решения задачи (1), (3), (4), (6), (7).

\section{3. Результаты численных экспериментов}

Приведем результаты решения следующих обратных задач.

Пример 1. Рассмотрим задачу

$$
\begin{gathered}
\frac{\partial v(x, t)}{\partial t}=a(x) \frac{\partial^{2} v(x, t)}{\partial x^{2}}+e^{t} C(x), \quad(x, t) \in \Omega=\{(x, t): 0<x<1,0<t \leq 1\}, \\
v(x, 0)=\cos 2 x+x \cos x, \quad v(x, 1)=e(\sin 2 x+x \cos x), \quad x \in[0,1], \\
v(0, t)=0, \quad v(1, t)=e^{t}(\sin 2+\cos 1), \quad t \in[0,1],
\end{gathered}
$$

где $a(x)=\cos x / 2 e^{x}$. Точным решением данной задачи являются функции:

$$
\begin{gathered}
C(x)=\sin 2 x+x \cos x+a(x)(4 \sin 2 x+2 \sin x+x \cos x), \\
v(x, t)=e^{t}(\sin 2 x+x \cos x) .
\end{gathered}
$$

Численные эксперименты проводились при различных количествах $N$ прямых $x=x_{i}, i=1, \ldots, N$. В Таблице 1 приведены результаты решения задачи при количестве прямых $N=10,20,30$. Для решения вспомогательных задач Коши использовался метод Рунге-Кутта четвертого порядка при различных шагах $h_{t}$. Были проведены расчеты при наличии случайных помех в функции $v(x, 1)$, которые определялись следующим образом:

$$
v^{\sigma}(x, 1)=v(x, 1)(1+\sigma \text { rand }),
$$

где $\sigma$ - процент уровня шума, rand - случайные числа, произведенные от равномерного распределения в интервале $[-1,1]$. Эти случайные числа получены с использованием функции MATLAB rand.

В Таблице 2 приведены результаты решения примера 1 при $N=20, h_{t}=0.001$ для уровней шума равных $\sigma=1 \%, \sigma=2 \%$ и $\sigma=3 \%$, а также без наличия шума, т.е. $\sigma=0 \%$. На Рисунке 1 даны графики точного (аналитическое решение) и полученного численными методами (приведенными в разд. 2) коэффициента $C(x)$ при различных уровнях шума $\sigma$ на основе данных Таблице 2 для примера 1.

Пример 2. Рассмотрим задачу

$\frac{\partial v(x, t)}{\partial t}=a(x) \frac{\partial^{2} v(x, t)}{\partial x^{2}}+e^{2 t+x} C(x), \quad(x, t) \in \Omega=\{(x, t): 0<x<1,0<t \leq 1\}$, 
Таблица 1: Полученные в результате расчетов и точные значения $C(x)$ для примера 1 при различном количестве прямых

\begin{tabular}{|l|l|l|l|l|l|l|l|}
\hline \multirow{2}{*}{$x_{i}$} & \multicolumn{2}{|l|}{$N=10$} & \multicolumn{2}{l|}{$N=20$} & \multicolumn{2}{l|}{$N=30$} & \multirow{2}{*}{$\begin{array}{l}\text { Toчное } \\
\end{array}$} \\
\cline { 2 - 8 } & $C\left(x_{i}\right)$ & $\left|\Delta C\left(x_{i}\right)\right|$ & $C\left(x_{i}\right)$ & $\left|\Delta C\left(x_{i}\right)\right|$ & $C\left(x_{i}\right)$ & $\left|\Delta C\left(x_{i}\right)\right|$ & \\
\hline 0.10 & 0.78920 & 0.00138 & 0.79023 & 0.00034 & 0.79042 & 0.00015 & 0.79057 \\
\hline 0.20 & 1.44602 & 0.00241 & 1.44783 & 0.00060 & 1.44817 & 0.00027 & 1.44843 \\
\hline 0.30 & 1.95795 & 0.00309 & 1.96027 & 0.00077 & 1.96070 & 0.00034 & 1.96104 \\
\hline 0.40 & 2.32230 & 0.00344 & 2.32488 & 0.00086 & 2.32536 & 0.00038 & 2.32574 \\
\hline 0.50 & 2.54452 & 0.00350 & 2.54715 & 0.00088 & 2.54764 & 0.00039 & 2.54803 \\
\hline 0.60 & 2.63616 & 0.00333 & 2.63866 & 0.00083 & 2.63912 & 0.00037 & 2.63949 \\
\hline 0.70 & 2.61277 & 0.00298 & 2.61501 & 0.00075 & 2.61543 & 0.00033 & 2.61576 \\
\hline 0.80 & 2.49205 & 0.00253 & 2.49395 & 0.00063 & 2.49430 & 0.00028 & 2.49458 \\
\hline 0.90 & 2.29217 & 0.00203 & 2.29369 & 0.00051 & 2.29397 & 0.00023 & 2.29420 \\
\hline
\end{tabular}

Таблица 2: Значения коэффициента $C(x)$ для примера 1 при различных уровнях шума $\sigma$

\begin{tabular}{|c|c|c|c|c|c|c|c|c|c|c|}
\hline \multirow{4}{*}{$i$} & \multirow{4}{*}{$x_{i}$} & \multicolumn{9}{|c|}{ Значение $C(x)$} \\
\hline & & \multirow{3}{*}{$\begin{array}{l}\text { Точное } \\
C\left(x_{i}\right)\end{array}$} & \multicolumn{8}{|c|}{ Полученное значение для $\sigma(\%)$} \\
\hline & & & \multicolumn{2}{|l|}{$\sigma=0.0$} & \multicolumn{2}{|l|}{$\sigma=1.0$} & \multicolumn{2}{|l|}{$\sigma=2.0$} & \multicolumn{2}{|l|}{$\sigma=3.0$} \\
\hline & & & $C\left(x_{i}\right)$ & $|\Delta C(\sigma)|$ & $C\left(x_{i}\right)$ & $|\Delta C(\sigma)|$ & $C\left(x_{i}\right)$ & $|\Delta C(\sigma)|$ & $C\left(x_{i}\right)$ & $|\Delta C(\sigma)|$ \\
\hline 1 & 0.05 & 0.41067 & 0.41048 & 0.00018 & 0.25370 & 0.15696 & 0.09693 & 0.31374 & -0.05985 & 0.47052 \\
\hline 2 & 0.10 & 0.79057 & 0.79023 & 0.00034 & 0.69303 & 0.09755 & 0.59582 & 0.19475 & 0.49862 & 0.29195 \\
\hline 3 & 0.15 & 1.13713 & 1.13664 & 0.00049 & 1.11625 & 0.02088 & 1.09586 & 0.04127 & 1.07547 & 6166 \\
\hline 4 & 0.20 & 1.44843 & 1.44783 & 0.00060 & 1.50566 & 0.05722 & 1.56348 & 0.11505 & 1.62131 & 0.17288 \\
\hline 5 & 0.25 & 1.72327 & 1.72257 & 0.00070 & 1.84539 & 0.12212 & 1.96821 & 0.24493 & 2.09102 & 0.36775 \\
\hline 6 & 0.30 & 1.96104 & 1.96027 & 0.00077 & 2.12392 & 0.16288 & 2.28758 & 0.32654 & 2.45123 & 0.49019 \\
\hline 7 & 0.35 & 2.16170 & 2.16088 & 0.00083 & 2.33561 & 0.17390 & 2.51034 & 0.34863 & 2.68507 & 0.52336 \\
\hline 8 & 0.40 & 2.32574 & 2.32488 & 0.00086 & 2.48110 & 0.15536 & 2.63733 & 0.31159 & 2.79355 & 0.46781 \\
\hline 9 & 0.45 & 2.45408 & 2.45320 & 0.00088 & 2.56670 & 0.11262 & 2.68019 & 0.22611 & 2.79369 & 0.33960 \\
\hline 10 & 0.50 & 2.54803 & 2.54715 & 0.00088 & 2.60275 & 0.05472 & 2.65834 & 0.11031 & 2.71393 & 0.16590 \\
\hline 11 & 0.55 & 2.60921 & 2.60835 & 0.00086 & 2.60163 & 0.00757 & 2.59492 & 0.01429 & 2.58821 & 0.02100 \\
\hline 12 & 0.60 & 2.63949 & 2.63866 & 0.00083 & 2.57563 & 0.06386 & 2.51261 & 0.12688 & 2.44958 & 0.18991 \\
\hline 13 & 0.65 & 2.64094 & 2.64015 & 0.00079 & 2.53506 & 0.10588 & 2.42998 & 0.21096 & 2.32490 & 0.31605 \\
\hline 14 & 0.70 & 2.61576 & 2.61501 & 0.00075 & 2.48706 & 0.12870 & 2.35910 & 0.25666 & 2.23115 & 0.38461 \\
\hline 15 & 0.75 & 2.56621 & 2.56551 & 0.00069 & 2.43503 & 0.13118 & 2.30455 & 0.26166 & 2.17406 & 0.39214 \\
\hline 16 & 0.80 & 2.49458 & 2.49395 & 0.00063 & 2.37894 & 0.11565 & 2.26392 & 0.23066 & 2.14891 & 0.34568 \\
\hline 17 & 0.85 & 2.40317 & 2.40260 & 0.00057 & 2.31608 & 0.08709 & 2.22955 & 0.17362 & 2.14303 & 0.26014 \\
\hline 18 & 0.90 & 2.29420 & 2.29369 & 0.00051 & 2.24230 & 0.05190 & 2.19090 & 0.10329 & 2.13951 & 0.15469 \\
\hline 19 & 0.95 & 2.16980 & 2.16936 & 0.00044 & 2.15325 & 0.01655 & 2.13715 & 0.03265 & 2.12105 & 0.04876 \\
\hline
\end{tabular}




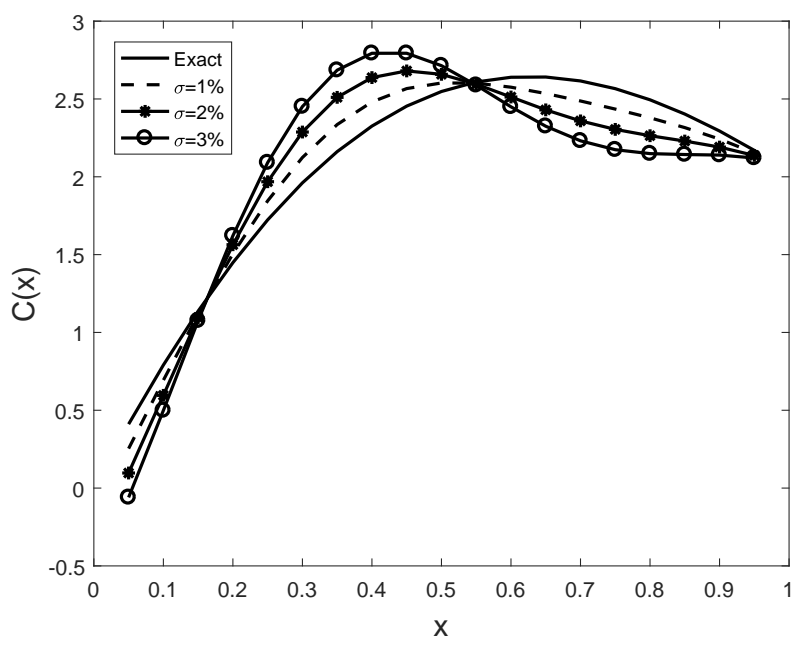

Puс. 1: Графики точного (Exact) и полученного численными методами коэффиииента $C(x)$ при различных уровнях шума для примера 1

$$
\begin{gathered}
v(x, 0)=e^{x}\left(\sin x+x^{3}\right), \quad v(x, 1)=e^{x+2}\left(\sin x+x^{3}\right), \quad x \in[0,1], \\
v(0, t)=0, \quad v(1, t)=e^{2 t+1}(\sin 1+1), \quad t \in[0,1],
\end{gathered}
$$

где $a(x)=e^{x} /\left(x^{2}+3\right)$. Точным решением данной задачи являются функции:

$$
\begin{gathered}
C(x)=2\left(\sin x+x^{3}\right)-a(x)\left(2 \cos x+x^{3}+6 x^{2}+6 x\right), \\
v(x, t)=e^{2 t+x}\left(\sin x+x^{3}\right) .
\end{gathered}
$$

В Таблице 3 приведены результаты решения задачи при числе прямых $N=10,20,30$. В Таблице 4 приведены результаты решения примера 2 при $N=20$, $h_{t}=0.001$ для уровней шума равных $\sigma=1 \%, \sigma=2 \%$ и $\sigma=3 \%$, а также без наличия шума, т.е. $\sigma=0 \%$. На Рис. 2 даны графики точного (аналитическое решение) и полученного численными методами коэффициента $C(x)$ при различных уровнях шума $\sigma$ на основе данных Таблице 4 для примера 2.

Точность решения обратных задач, как и следовало ожидать, существенно зависит от числа используемых прямых $N$ в методе прямых для аппроксимации исходной краевой задачи.

В задаче определения $C(x)$ увеличение числа прямых приводит к увеличению порядка системы дифференциальных уравнений с обыкновенными производными, равной $N^{2}$. Это вызывает существенное увеличение объема вычислений, а следовательно и увеличение погрешности вычислительного характера. Поэтому при решении конкретной задачи идентификации коэффициента $C(x)$ для выбора числа прямых необходимо провести дополнительный численный анализ.

\section{Заключение}

Предлагаемые в работе численные методы решения задач параметрической идентификации для параболического уравнения целесообразны, поскольку они 
Таблица 3: Полученные в результате расчетов и точные значения $C(x)$ для примера 2 при различном количестве прямых

\begin{tabular}{|c|c|c|c|c|c|c|c|}
\hline \multirow{2}{*}{$x_{i}$} & \multicolumn{2}{|l|}{$N=10$} & \multicolumn{2}{|l|}{$N=20$} & \multicolumn{2}{|l|}{$N=30$} & \multirow{2}{*}{$\begin{array}{l}\text { Точное } \\
C\left(x_{i}\right)\end{array}$} \\
\hline & $C\left(x_{i}\right)$ & $\left|\Delta C\left(x_{i}\right)\right|$ & $C\left(x_{i}\right)$ & $\left|\Delta C\left(x_{i}\right)\right|$ & $C\left(x_{i}\right)$ & $\left|\Delta C\left(x_{i}\right)\right|$ & \\
\hline 0.10 & -0.78007 & 0.00837 & -0.77379 & 0.00209 & -0.77262 & 0.00093 & -0.77169 \\
\hline 0.20 & -0.96633 & 0.01036 & -0.95856 & 0.00259 & -0.95712 & 0.00115 & -0.95597 \\
\hline 0.30 & -1.23631 & 0.01266 & -1.22681 & 0.00316 & -1.22506 & 0.00140 & -1.22365 \\
\hline 0.40 & -1.59458 & 0.01529 & -1.58310 & 0.00382 & -1.58098 & 0.00170 & -1.57928 \\
\hline 0.50 & -2.04609 & 0.01829 & -2.03237 & 0.00457 & -2.02983 & 0.00203 & -2.02780 \\
\hline 0.60 & -2.59630 & 0.02166 & -2.58005 & 0.00541 & -2.57704 & 0.00240 & -2.57464 \\
\hline 0.70 & -3.25138 & 0.02544 & -3.23230 & 0.00635 & -3.22877 & 0.00282 & -3.22594 \\
\hline 0.80 & -4.01853 & 0.02965 & -3.99629 & 0.00741 & -3.99217 & 0.00328 & -3.98888 \\
\hline 0.90 & -4.90636 & 0.03432 & -4.88060 & 0.00856 & -4.87596 & 0.00392 & -4.87204 \\
\hline
\end{tabular}

Таблица 4: Значения коэффициента $C(x)$ для примера 2 при различных уровнях шума $\sigma$

\begin{tabular}{|c|c|c|c|c|c|c|c|c|c|c|}
\hline \multirow{4}{*}{$i$} & \multirow{4}{*}{$x_{i}$} & \multicolumn{9}{|c|}{ Значение $C(x)$} \\
\hline & & \multirow{3}{*}{$\begin{array}{l}\text { Точное } \\
C\left(x_{i}\right)\end{array}$} & \multicolumn{8}{|c|}{ Полученное значение для $\sigma(\%)$} \\
\hline & & & \multicolumn{2}{|l|}{$\sigma=0.0$} & \multicolumn{2}{|l|}{$\sigma=1.0$} & \multicolumn{2}{|l|}{$\sigma=2.0$} & \multicolumn{2}{|l|}{$\sigma=3.0$} \\
\hline & & & $C\left(x_{i}\right)$ & $\Delta C(\sigma)$ & $C\left(x_{i}\right)$ & $\Delta C(\sigma)$ & $C\left(x_{i}\right)$ & $\mid \Delta C(\sigma)$ & $C\left(x_{i}\right)$ & $\Delta C(\sigma)$ \\
\hline 1 & 0.05 & -0.70952 & -0.71139 & 0.00187 & -0.75498 & 0.04546 & -0.79857 & 0.08905 & -0.84216 & 0.13264 \\
\hline 2 & 0.10 & -0.77169 & -0.77379 & 0.00209 & -0.81103 & 0.03933 & -0.84827 & 0.07658 & -0.88551 & 0.11382 \\
\hline 3 & 0.15 & -0.85368 & -0.85601 & 0.00233 & -0.87777 & 0.02409 & -0.89953 & 0.04586 & -0.92130 & 0.06762 \\
\hline 4 & 0.20 & -0.95597 & -0.95856 & 0.00259 & -0.95626 & 0.00029 & -0.95396 & 0.00201 & -0.95167 & 0.00430 \\
\hline 5 & 0.25 & -1.07911 & -1.08197 & 0.00286 & -1.04885 & 0.03026 & -1.01572 & 0.06339 & -0.98259 & 0.09652 \\
\hline 6 & 0.30 & -1.22365 & -1.22681 & 0.00316 & -1.15924 & 0.06441 & -1.09167 & 0.13198 & -1.02410 & 0.19955 \\
\hline 7 & 0.35 & -1.39017 & -1.39365 & 0.00348 & -1.29247 & 0.09771 & -1.19128 & 0.19889 & -1.09010 & 0.30008 \\
\hline 8 & 0.40 & -1.57928 & -1.58310 & 0.00382 & -1.45459 & 0.12469 & -1.32608 & 0.25320 & -1.19757 & 0.38172 \\
\hline 9 & 0.45 & -1.79160 & -1.79579 & 0.00418 & -1.65225 & 0.13935 & -1.50871 & 0.28289 & -1.36518 & 0.42643 \\
\hline 10 & 0.50 & -2.02780 & -2.03237 & 0.00457 & -1.89195 & 0.13584 & -1.75154 & 0.27626 & -1.61113 & 0.41667 \\
\hline 11 & 0.55 & -2.28856 & -2.29354 & 0.00498 & -2.17918 & 0.10938 & -2.06483 & 0.22374 & -1.95047 & 0.33810 \\
\hline 12 & 0.60 & -2.57464 & -2.58005 & 0.00541 & -2.51739 & 0.05724 & -2.45474 & 0.11990 & -2.39208 & 0.18255 \\
\hline 13 & 0.65 & -2.88681 & -2.89268 & 0.00587 & -2.90706 & 0.02024 & -2.92143 & 0.03462 & -2.93580 & 0.04899 \\
\hline 14 & 0.70 & -3.22594 & -3.23230 & 0.00635 & -3.34488 & 0.11894 & -3.45747 & 0.23153 & -3.57006 & 0.34412 \\
\hline 15 & 0.75 & -3.59296 & -3.59983 & 0.00687 & -3.82344 & 0.23048 & -4.04706 & 0.45410 & -4.27067 & 0.67771 \\
\hline 16 & 0.80 & -3.98888 & -3.99629 & 0.00741 & -4.33128 & 0.34239 & -4.66626 & 0.67738 & -5.00125 & 1.01236 \\
\hline 17 & 0.85 & -4.41483 & -4.42281 & 0.00798 & -4.85371 & 0.43888 & -5.28462 & 0.86979 & -5.71552 & 1.30069 \\
\hline 18 & 0.90 & -4.87204 & -4.88060 & 0.00856 & -5.37432 & 0.50228 & -5.86804 & 0.99600 & -6.36176 & 1.48972 \\
\hline 19 & 0.95 & -5.36187 & -5.37108 & 0.00921 & -5.87711 & 0.51524 & -6.38314 & 1.02127 & -6.88917 & 1.52730 \\
\hline
\end{tabular}




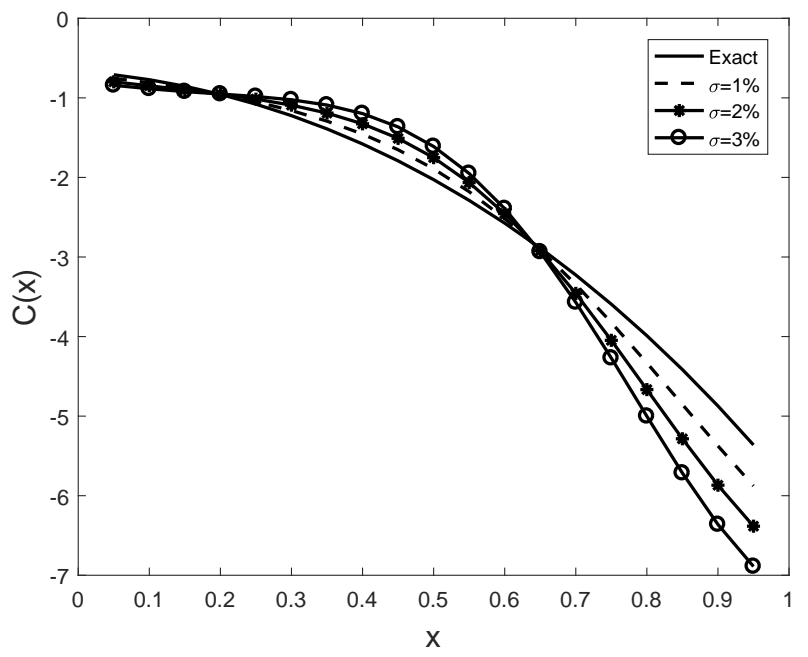

Pис. 2: Графики точного (Eхасt) и полученного численными методами коэфбиииента $C(x)$ при различных уровнях шума для примера 2

приводятся к решению вспомогательных, хорошо исследованных задач Коши и не требуют построения итерационных процедур. Для этого может быть использовано стандартное программное обеспечение, в частности MATLAB. K рассмотренным классам задач сводятся часто встречающиеся на практике задачи с нелокальными начальными и граничными условиями.

Важно отметить, что предлагаемая методика построения численных методов может быть использована для других типов уравнений с частными производными с другими заданными видами начально-краевых условий.

Автор выражает искреннюю благодарность проф. К.Р. Айда-заде за ценные советы и внимание к работе.

\section{Список литературы}

[1] Cannon J.R., Duchateau P. Structural identification of an unknown source term in a heat equation // Inverse Problems. 1998. Vol. 14. Pp. 535-551.

[2] Fatullayev A.G. Numerical solution of the inverse problem of determining an unknown source term in a two-dimensional heat equation // Applied Mathematics and Computation. 2004. Vol. 152. Pp. 659-666.

[3] Liu C.-S. An two-stage LGSM to identify time dependent heat source through an internal measurement of temperature // International Journal of Heat and Mass Transfer. 2009. Vol. 52. Pp. 1635-1642.

[4] Liu C.-S. A Lie-group shooting method for reconstructing a past time-dependent heat source // International Journal of Heat and Mass Transfer. 2012. Vol. 55. Pp. 1773-1781. 
[5] Yang L., Deng Z.-C., Yu J.-N., Luo G.-W. Optimization method for the inverse problem of reconstructing the source term in a parabolic equation // Mathematics and Computers in Simulation. 2009. Vol. 80. Pp. 314-326.

[6] Farcas A., Lesnic D. The boundary-element method for the determination of a heat source dependent on one variable // Journal of Engineering Mathematics. 2006. Vol. 54. Pp. 375-388.

[7] Ling L., Yamamoto M., Hon Y.C. Identification of source locations in twodimensional heat equations // Inverse Problems. 2006. Vol. 22. Pp. 1289-1305.

[8] Mohebbia A., Abbasia M. A fourth-order compact difference scheme for the parabolic inverse problem with an overspecification at a point // Inverse Problems in Science and Engineering. 2015. Vol. 23, № 3. Pp. 457-478.

[9] Johansson T., Lesnic D. A variational method for identifying a spacewisedependent heat source // IMA Journal of Applied Mathematics. 2007. Vol. 72. Pp. 748-760.

[10] Hasanov A. Identification of spacewise and time dependent source terms in 1D heat conduction equation from temperature measurement at a final time // International Journal of Heat and Mass Transfer. 2012. Vol. 55. Pp. 2069-2080.

[11] Hasanov A. An inverse source problem with single Dirichlet type measured output data for a linear parabolic equation // Applied Mathematics Letters. 2011. Vol. 24. Pp. 1269-1273.

[12] Прилепко А.И., Костин А.Б. О некоторых обратных задачах для параболических уравнений с финальным и интегральным наблюдением // Математический сборник. 1992. Т. 183, № 4. С. 49-68.

[13] Саватеев Е.Г. О задаче идентификации коэффициента параболического уравнения // Сибирский математический журнал. 1995. Т. 36, № 1. С. 177-185.

[14] Ivanchov M.I. The inverse problem of determining the heat source power for a parabolic equation under arbitrary boundary conditions // Journal of Mathematical Sciences. 1998. Vol. 88, № 3. Pp. 432-436.

[15] Yan L., Fu C.L., Yang F.L. The method of fundamental solutions for the inverse heat source problem // Engineering Analysis with Boundary Elements. 2008. Vol. 32. Pp. 216-222.

[16] Ahmadabadi M.N., Arab M., Maalek Ghaini F.M. The method of fundamental solutions for the inverse space-dependent heat source problem // Engineering Analysis with Boundary Elements. 2009. Vol. 33. Pp. 1231-1235.

[17] Ismailov M.I., Kanca F., Lesnic D. Determination of a time-dependent heat source under nonlocal boundary and integral overdetermination conditions // Applied Mathematics and Computation. 2011. Vol. 218. Pp. 4138-4146. 
[18] Пулькина Л.С. Об одном классе нелокальных задач и их связи с обратными задачами // Труды Третьей Всероссийской научной конференции «Математическое моделирование и краевые задачи». Ч. 3: Дифференциальные уравнения и краевые задачи. Самара: СамГТУ, 2006. С. 190-192.

[19] Камынин В.Л. Об обратной задаче определения правой части в параболическом уравнении с условием интегрального переопределения // Математические заметки. 2005. Т. 77, № 4. С. 522-534.

[20] Прилепко А.И., Ткаченко Д.С. Корректность обратной задачи об источнике для параболических систем // Дифференциальные уравнения. 2004. Т. 40, № 11. C. $1540-1547$.

[21] Нахушев А.М. Уравнения математической биологии. М.: Высшая школа, 1995. $305 \mathrm{c}$.

[22] Самарский А.А. О некоторых проблемах современной теории дифференциальных уравнений // Дифференциальные уравнения. 1980. Т. 16, № 11. C. $1221-1228$.

[23] Ионкин Н.И. Решение одной краевой задачи теории теплопроводности с неклассическим краевым условием // Дифференциальные уравнения. 1977. T. 13, № 2. C. 294-304.

[24] Bouziani A., Benouar N-E. Probleme mixte avec conditions integrales pour une classe d'equations paraboliques // Comptes Rendus de l'Academie des Sciences Series I - Mathematics. 1995. Vol. 321. Pp. 1177-1182.

[25] Водахова В.А. Краевая задача с нелокальным условием А.М. Нахушева для одного псевдопараболического уравнения влагопереноса // Дифференциальные уравнения. 1982. Т. 18, № 2. С. 280-285.

[26] Белавин В.А., Капица С.П., Курдюмов С.П. Математическая модель глобальных демографических процессов с учетом пространственного распределения // Журнал вычислительной математики и математической физики. 1998. T. 38, № 6. C. 885-902.

[27] Schiesser W.E. The Numerical Method of Lines: Integration of Partial Differential Equations. San Diego: Academic Press, 1991. 326 p.

[28] Самусенко А.В., Фролова С.В. Многоточечные схемы продольного варианта метода прямых повышенной точности для решения некоторых задач математической физики // Известия Национальной академии наук Беларуси. Серия физико-математических наук. 2009. № 3. С. 31-39.

[29] Лисковец О.А. Метод прямых // Дифференциальные уравнения. 1965. Т. 1, № 12 . С. $1662-1678$.

[30] Aida-zade K.R., Rahimov A.B. An approach to numerical solution of some inverse problems for parabolic equations // Inverse Problems in Science and Engineering. 2014. Vol. 22, № 1. Pp. 96-111. 
[31] Будак Б.М. О методе прямых для некоторых квазилинейных краевых задач параболического типа // Журнал вычислительной математики и математической физики. 1961. Т. 1, № 6. С. 1105-1112.

\section{Образец цитирования}

Рагимов А.Б. К численному решению обратных задач для линейного параболического уравнения // Вестник ТвГУ. Серия: Прикладная математика. 2018. № 1. C. 69-87. https://doi.org/10.26456/vtpmk162

\section{Сведения об авторах}

\section{1. Рагимов Анар Бейбала}

ведущий научный сотрудник Института систем управления НАН Азербайджана.

Азербайджан, AZ1141, г. Баку, ул. Б. Вахабзаде, д. 9, Институт систем управления НАНА. E-mail: anar_r@yahoo.com, anar.rahimov@fresnel.fr 


\title{
NUMERICAL SOLUTION TO INVERSE PROBLEMS FOR LINEAR PARABOLIC EQUATION
}

\author{
Rahimov Anar Beybala \\ Leading researcher at Institute of Control Systems, \\ Azerbaijan National Academy of Sciences \\ Azerbaijan, AZ1141, Baku, B. Vahabzadeh str. 9, \\ Institute of Control Systems of ANAS \\ E-mail: anar_r@yahoo.com,anar.rahimov@fresnel.fr
}

Received 21.09.2017, revised 01.03.2018.

In the paper, the inverse problems for a parabolic equation with unknown coefficients on the right-hand side are considered. Boundary value problems with nonlocal conditions are reduced to such problems. We investigate separately two cases: unknown coefficients depend on either time variable only or phase coordinates only. A numerical method to solve the problems by using the method of lines is suggested. The results of numerical experiments on test problems are given.

Keywords: inverse problem, nonlocal conditions, method of lines, parabolic equation, parametric identification.

\section{Bibliographic citation}

Rahimov A.B. Numerical solution to inverse problems for linear parabolic equation. Vestnik TvGU. Seriya: Prikladnaya Matematika [Herald of Tver State University. Series: Applied Mathematics], 2018, no. 1, pp. 69-87. (in Russian). https://doi.org/10.26456/vtpmk162

\section{Список литературы}

[1] Cannon J.R., Duchateau P. Structural identification of an unknown source term in a heat equation. Inverse Problems, 1998, vol. 14, pp. 535-551.

[2] Fatullayev A.G. Numerical solution of the inverse problem of determining an unknown source term in a two-dimensional heat equation. Applied Mathematics and Computation, 2004, vol. 152, pp. 659-666.

[3] Liu C.-S. An two-stage LGSM to identify time dependent heat source through an internal measurement of temperature. International Journal of Heat and Mass Transfer, 2009, vol. 52, pp. 1635-1642.

[4] Liu C.-S. A Lie-group shooting method for reconstructing a past time-dependent heat source. International Journal of Heat and Mass Transfer, 2012, vol. 55, pp. $1773-1781$. 
[5] Yang L., Deng Z.-C., Yu J.-N., Luo G.-W. Optimization method for the inverse problem of reconstructing the source term in a parabolic equation. Mathematics and Computers in Simulation, 2009, vol. 80, pp. 314-326.

[6] Farcas A., Lesnic D. The boundary-element method for the determination of a heat source dependent on one variable. Journal of Engineering Mathematics, 2006, vol. 54 , pp. $375-388$.

[7] Ling L., Yamamoto M., Hon Y.C. Identification of source locations in twodimensional heat equations. Inverse Problems, 2006, vol. 22, pp. 1289-1305.

[8] Mohebbia A., Abbasia M. A fourth-order compact difference scheme for the parabolic inverse problem with an overspecification at a point. Inverse Problems in Science and Engineering, 2015, vol. 23(3), pp. 457-478.

[9] Johansson T., Lesnic D. A variational method for identifying a spacewisedependent heat source. IMA Journal of Applied Mathematics, 2007, vol. 72, pp. $748-760$.

[10] Hasanov A. Identification of spacewise and time dependent source terms in 1D heat conduction equation from temperature measurement at a final time. International Journal of Heat and Mass Transfer, 2012, vol. 55, pp. 2069-2080.

[11] Hasanov A. An inverse source problem with single Dirichlet type measured output data for a linear parabolic equation. Applied Mathematics Letters, 2011, vol. 24, pp. 1269-1273.

[12] Prilepko A.I., Kostin A.B. On certain inverse problems for parabolic equations with final and integral observation. Sbornik: Mathematics, 1993, vol. 75(2), pp. $473-490$.

[13] Savateev E.G. On the problem of identification of a coefficient in a parabolic equation. Siberian Mathematical Journal, 1995, vol. 36(1), pp. 160-167.

[14] Ivanchov M.I. The inverse problem of determining the heat source power for a parabolic equation under arbitrary boundary conditions. Journal of Mathematical Sciences, 1998, vol. 88(3), pp. 432-436.

[15] Yan L., Fu C.L., Yang F.L. The method of fundamental solutions for the inverse heat source problem. Engineering Analysis with Boundary Elements, 2008, vol. 32, pp. 216-222.

[16] Ahmadabadi M.N., Arab M., Maalek Ghaini F.M. The method of fundamental solutions for the inverse space-dependent heat source problem. Engineering Analysis with Boundary Elements, 2009, vol. 33, pp. 1231-1235.

[17] Ismailov M.I., Kanca F., Lesnic D. Determination of a time-dependent heat source under nonlocal boundary and integral overdetermination conditions. Applied Mathematics and Computation, 2011, vol. 218, pp. 4138-4146. 
[18] Pulkina L.S. On one class of nonlocal problems and their connection with inverse problems. Trudy Tret'ei Vserossiiskoi nauchnoi konferentsii «Matematicheskoe modelirovanie i kraevye zadachi». Ch. 3: Differentsial'nye Uravneniya i kraevye zadachi [Proceedings of the Third All-Russia scientific conference «Mathematical modelling and boundary value problems», Part 3: Differential equations and boundary value problems], Samara: SSTU, 2006, pp. 190-192. (in Russian)

[19] Kamynin V.L. On the inverse problem of determining the right-hand side of a parabolic equation under an integral overdetermination condition. Mathematical Notes, 2005, vol. 77(4), pp. 482-493.

[20] Prilepko A.I., Tkachenko D.S. Well-posedness of the inverse source problem for parabolic systems. Differential Equations, 2004, vol. 40(11), pp. 1619-1626.

[21] Nakhushev A.M. Uravneniya Matematicheskoy Biologii [Equations of Mathematical Biology]. «Vysshaya Shkola» Publ., Moscow, 1995, 305 p. (in Russian)

[22] Samarskii A.A. On some problems of the modern theory of differential equations. Differentsialniye Uravneniya [Differential Equations], 1980, vol. 16(11), pp. 1221-1228. (in Russian)

[23] Ionkin N.I. Solution to one boundary-value thermal conductivity problem with nonclassical boundary condition. Differentsialniye Uravneniya [Differential Equations], 1977, vol. 13(2), pp. 294--304. (in Russian)

[24] Bouziani A., Benouar N-E. Probleme mixte avec conditions integrales pour une classe d'equations paraboliques. Comptes Rendus de l'Academie des Sciences Series I - Mathematics, 1995, vol. 321, pp. 1177-1182.

[25] Vodakhova V.A. A.M. Nahushev's boundary-value problem with nonlocal condition for one pseudo-parabolic moisture transfer equation. Differentsialniye Uravneniya [Differential Equations], 1982, vol. 18(2). pp. 280--285. (in Russian)

[26] Belavin V.A., Kapitsa S.P., Kurdyumov S.P. Mathematical model of demographic processes with regard of a space distribution. Zhyrnal Vychislitelnoy Matematiki i Matematicheskoy Fiziki [Computational Mathematics and Mathematical Physics], 1998, vol. 38(6), pp. 885-902. (in Russian)

[27] Schiesser W.E. The Numerical Method of Lines: Integration of Partial Differential Equations. San Diego: Academic Press, 1991. 326 p.

[28] Samusenko A.V., Frolova S.V. Multipoint schemes of the longitudinal variant of highly accurate method of lines to solve some problems of mathematical physics. Izvestiya Natsionalnoy Akademii Nauk Belorusi. Seriya Fiziko-Matematicheskikh Nauk [Proceedings of the National Academy of Sciences of Belarus. Series of Physical-Mathematical Sciences], 2009, no. 3, pp. 31--39. (in Russian)

[29] Liskovets O.A. The method of lines. Differentsialniye Uravneniya [Differential Equations], 1965, vol. 1(12), pp. 1662-1678. (in Russian) 
[30] Aida-zade K.R., Rahimov A.B. An approach to numerical solution of some inverse problems for parabolic equations. Inverse Problems in Science and Engineering, 2014, vol. 22(1), pp. 96-111.

[31] Budak B.M. The method of lines for some quasilinear parabolic boundaryvalue problems. Zhyrnal Vychislitelnoy Matematiki $i$ Matematicheskoy Fiziki [Computational Mathematics and Mathematical Physics], 1961, vol. 1(6), pp. 1105-1112. (in Russian) 